\title{
Elevated serum levels of visfatin in gestational diabetes: a comparative study across various degrees of glucose tolerance
}

\author{
K. C. Lewandowski • N. Stojanovic • M. Press • \\ S. M. Tuck $\cdot$ K. Szosland $\cdot$ M. Bienkiewicz $\cdot$ M. Vatish • \\ A. Lewinski • G. M. Prelevic • H. S. Randeva
}

Received: 23 November 2006 / Accepted: 20 December 2006 / Published online: 2 March 2007

(C) Springer-Verlag 2007

\begin{abstract}
Aims/hypothesis Concentrations of visfatin are increased in insulin-resistant conditions, but the relationship between visfatin and insulin and/or insulin resistance indices in pregnancy remains unclear. Insulin resistance in pregnancy is further accentuated in women with gestational diabetes mellitus (GDM). Thus we assessed serum levels of visfatin
\end{abstract}

Electronic supplementary material The online version of this article (doi:10.1007/s00125-007-0610-7) contains supplementary material, which is available to authorised users.

G. M. Prelevic and H. S. Randeva are both senior authors.

K. C. Lewandowski $\cdot$ K. Szosland - A. Lewinski

Department of Endocrinology and Metabolic Diseases,

The Medical University of Lodz

and Polish Mother's Memorial Research Institute,

Lodz, Poland

N. Stojanovic

Department of Diabetes, Oldchurch Hospital,

Essex, UK

\section{Bienkiewicz}

Department of Quality Control and Radiation Protection,

The Medical University of Lodz,

Lodz, Poland

\section{Press · G. M. Prelevic}

Department of Endocrinology, Royal Free Hospital,

London, UK

K. C. Lewandowski $\cdot$ M. Vatish $\cdot H$. S. Randeva $(\bowtie)$

Molecular Medicine Group, Department of Biological Sciences,

The University of Warwick,

Coventry CV4 7AL, UK

e-mail: hrandeva@bio.warwick.ac.uk

S. M. Tuck

Department of Obstetrics, Royal Free Hospital,

London, UK in pregnant women with varying degrees of glucose tolerance.

Materials and methods Fasting visfatin levels were measured at 28 weeks of gestation in 51 women divided according to their response to a $50-\mathrm{g}$ glucose challenge test (GCT) and a 75-g OGTT: control subjects $(n=20)$ had normal responses to both a GCT and an OGTT; the intermediate group (IG; $n=15$ ) had a false-positive GCT, but a normal OGTT; the GDM group $(n=16)$ had abnormal GCTs and OGTTs.

Results There were no age or BMI differences between analysed groups. Across the subgroups there was a progressive increase in glucose and insulin at $120 \mathrm{~min}$ of the OGTT $(p<0.01)$. This was accompanied by an increase in visfatin, from $76.8 \pm 14.1 \mathrm{ng} / \mathrm{ml}$ in the control subjects, to $84.0 \pm 14.7 \mathrm{ng} / \mathrm{ml}$ in the IG group and $93.1 \pm 12.3 \mathrm{ng} / \mathrm{ml}$ in the GDM group ( $p<0.01$ for GDM vs control subjects). There was a positive correlation between visfatin and fasting insulin $(r=0.38, p=0.007)$ and insulin at $120 \mathrm{~min}$ of the OGTT ( $r=0.39, p=0.006)$.

Conclusions/interpretation An increase in fasting visfatin, the levels of which correlate with both fasting and postglucose-load insulin concentrations, accompanies worsening glucose tolerance in the third trimester of pregnancy. However, the significance of these findings, and in particular the role of visfatin in the regulation of insulin sensitivity during pregnancy, remains to be elucidated.

Keywords Gestational diabetes mellitus .

Glucose tolerance $\cdot$ Insulin resistance $\cdot$ Visfatin
Abbreviations
GCT glucose challenge test
GDM gestational diabetes mellitus 
HOMA homeostasis model assessment

IG intermediate group

IR insulin resistance

IRI insulin resistance index

\section{Introduction}

Visfatin, a $52 \mathrm{kDa}$ cytokine, also known as pre-beta cell colony enhancing factor, is highly expressed in visceral fat, and exerts insulin-mimicking effects through activation of an insulin receptor, although in a manner distinct from that of insulin [1]. The role of visfatin in human physiology and pathophysiology remains to be elucidated, while, according to some authors, plasma concentrations of visfatin are elevated in obesity [2] and type 2 diabetes [3], which are states characterised by insulin resistance (IR) and typically also observed in gestational diabetes mellitus (GDM). There are also, however, data pointing to possible lower visfatin levels in obese subjects [4], and to visfatin's role in NAD biosynthesis [5]. Therefore, we hypothesised that concentrations of visfatin may be altered in GDM, possibly as a result of increased IR, typical for GDM. Hence we examined serum concentrations of visfatin in women with various degrees of glucose intolerance in pregnancy.

\section{Subjects and methods}

In our practice, all women at 28 weeks of gestation are screened for GDM and are evaluated with a 50-g glucose challenge test (GCT). Women with plasma glucose $<7.8 \mathrm{mmol} / \mathrm{l}$ at $1 \mathrm{~h}$ after the GCT are regarded as normal and subjected to routine antenatal care. Women with plasma glucose $\geq 7.8 \mathrm{mmol} / 1$ after the GCT are subjected to a 75-g OGTT (for details of subjects and methods see the Electronic Supplementary Material [ESM]). GDM is diagnosed according to WHO criteria [6]. A cohort of 51 matched pregnant women was included in the present study and was taking no medication. Women were divided into three groups: matched control subjects $(n=20$, age $32 \pm$ 4.0 years, BMI $26 \pm 4.0 \mathrm{~kg} / \mathrm{m}^{2}$ [mean $\left.\pm \mathrm{SD}\right]$ ) had normal responses to both a GCT and an OGTT; an intermediate group (IG) $\left(n=15\right.$, age $33 \pm 4.0$ years, BMI $\left.27 \pm 4.2 \mathrm{~kg} / \mathrm{m}^{2}\right)$ had a false-positive GCT, but normal OGTT; while the GDM group ( $n=16$, age $33 \pm 3.9$ years, BMI $28 \pm 4.1 \mathrm{~kg} / \mathrm{m}^{2}$ ) had both abnormal GCTs and OGTTs (see ESM).

Our Ethics Committee approved our study, and having obtained informed consent we measured serum levels of fasting visfatin as well as glucose and insulin during the OGTT, with samples taken onto ice immediately, centrifuged and stored at $-80^{\circ} \mathrm{C}$. Serum visfatin was measured by an ELISA kit (Phoenix Pharmaceuticals, Burlingame, $\mathrm{CA}$, USA; $\mathrm{CV}<6 \%$ ). Insulin was measured by ELISA (DakoCytomation Ltd, Ely, Cambs, UK).

IR was assessed by homeostasis model assessment (HOMA) [7] (where HOMA=[fasting insulin (pmol/1)] $\times$ [fasting glucose $(\mathrm{mmol} / \mathrm{l})] / 22.5$, i.e. with insulin expressed in SI units ( $\mathrm{pmol} / \mathrm{l}$ instead of $\mathrm{mU} / \mathrm{ml}$ ) and the insulin resistance index (IRI) [8] (see ESM).

Statistical analysis The data were analysed by means of simple descriptive statistics and non-parametric tests of significance: the Mann-Whitney $U$ test for comparison of distributions in two independent groups and the Kruskal-Wallis in the case of more than two groups (see ESM). In all analyses, statistical significance was considered achieved at a value of $p \leq 0.05$. All the calculations were derived by means of Statistica v6.0 software.

\section{Results}

Results of the study are summarised in Table 1 and Fig. 1, as well as in ESM Table 1 and Fig. 1. There was no evidence of pregnancy-related complications at the time of the study. There were no differences in age or BMI between the analysed groups (ESM Table 1). All women with GDM had glucose levels at $120 \mathrm{~min}$ of the OGTT $>7.8 \mathrm{mmol} / \mathrm{l}$. There was an increase in fasting insulin and HOMA index in both IG and GDM groups in comparison with the normal glucose tolerance control subjects $(p<0.01)$; however, there were no statistical differences in fasting insulin, fasting glucose and HOMA between the IG and GDM groups ( $p>$ 0.10 ; Table 1). In contrast, there was no difference in IRI between control and IG groups $(p=0.23)$. There was, however, a marked difference in the value of the IRI between the GDM and IG $(p=0.015)$, and between the GDM and control group ( $p<0.001$; Table 1).

Worsening of glucose tolerance was accompanied by an increase in fasting visfatin, from $76.8 \pm 14.1 \mathrm{ng} / \mathrm{ml}$ in control subjects, to $84.0 \pm 14.7 \mathrm{ng} / \mathrm{ml}$ in $\mathrm{IG}$ and $93.1 \pm$ $12.3 \mathrm{ng} / \mathrm{ml}$ in the GDM group ( $p<0.01$ for GDM vs control subjects, with a trend towards higher visfatin levels for IG vs control subjects $[p=0.07]$ and GDM vs IG $[p=0.08]$ ) (Table 1, Fig. 1). There were positive correlations between serum visfatin concentrations and fasting insulin $\left(r_{\mathrm{s}}=0.38\right.$, $p=0.007)$, insulin levels at $120 \mathrm{~min}$ of the OGTT $\left(r_{\mathrm{s}}=0.39\right.$; $p=0.006$ ) (ESM Fig. 1a,b), glucose at $120 \mathrm{~min}$ of the OGTT $\left(r_{\mathrm{s}}=0.35 ; p=0.012\right)$, HOMA $\left(r_{\mathrm{s}}=0.35, p=0.012\right)$ and IRI $\left(r_{\mathrm{s}}=0.32, p=0.02\right)$. In the multiple regression model the covariates including fasting insulin, insulin at $120 \mathrm{~min}$ of the OGTT and glucose at $120 \mathrm{~min}$ of the OGTT explained only $18 \%$ of variability of serum visfatin concentration. 
Table 1 Descriptive statistics for the levels of insulin and glucose parameters as well as HOMA and IRI in the three groups of women: control; intermediate (i.e. false-positive GCT); and GDM

\begin{tabular}{|c|c|c|c|c|c|c|c|c|c|}
\hline Variable and group & $n$ & Mean & $95 \% \mathrm{CI}$ & Median & SD & Minimum & Maximum & $\begin{array}{l}\text { Differences } \\
\text { between groups }\end{array}$ & $p$ value $^{\mathrm{a}}$ \\
\hline \multicolumn{10}{|c|}{ Glucose: OGTT $0 \mathrm{~min}(\mathrm{mmol} / \mathrm{l})$} \\
\hline Control & 20 & 4.1 & $3.9-4.3$ & 4.1 & 0.4 & 3.6 & 4.9 & & \multirow{3}{*}{0.03} \\
\hline Intermediate & 15 & 4.2 & $4.1-4.4$ & 4.2 & 0.3 & 3.7 & 4.7 & & \\
\hline GDM & 16 & 4.6 & $4.1-5.1$ & 4.4 & 1.0 & 3.6 & 8.0 & $* * \mathrm{C}$ & \\
\hline \multicolumn{10}{|c|}{ Glucose: OGTT $120 \mathrm{~min}(\mathrm{mmol} / \mathrm{l})$} \\
\hline Control & 20 & 5.7 & $5.1-6.3$ & 5.7 & 1.2 & 3.7 & 7.7 & & \multirow{3}{*}{0.0001} \\
\hline Intermediate & 15 & 6.4 & $5.8-7.0$ & 6.7 & 1.0 & 4.4 & 7.6 & & \\
\hline GDM & 16 & 9.5 & $8.9-10.2$ & 9.6 & 1.0 & 8.0 & 11.6 & $* * * \mathrm{C}, * * * \mathrm{IN}$ & \\
\hline \multicolumn{10}{|c|}{ Insulin: OGTT $0 \mathrm{~min}(\mathrm{pmol} / \mathrm{l})$} \\
\hline Control & 20 & 36.7 & $25.8-47.5$ & 31.2 & 232 & 14.4 & 118.2 & & \multirow{3}{*}{0.0012} \\
\hline Intermediate & 15 & 63.8 & $42.1-85.6$ & 50.8 & 39.2 & 25.1 & 163.3 & $* * \mathrm{C}$ & \\
\hline GDM & 16 & 61.2 & $48.2-74.2$ & 56.8 & 24.4 & 22.9 & 119.5 & $* * * \mathrm{C}$ & \\
\hline \multicolumn{10}{|c|}{ Insulin: OGTT $120 \mathrm{~min}(\mathrm{pmol} / \mathrm{l})$} \\
\hline Control & 20 & 303.7 & $238.8-368.5$ & 274.2 & 138.5 & 95.3 & 566.5 & & \multirow{3}{*}{0.0003} \\
\hline Intermediate & 15 & 485.3 & $334.1-636.4$ & 417.2 & 261.8 & 106.1 & 1081.6 & ${ }^{*} \mathrm{C}$ & \\
\hline GDM & 16 & 719.8 & $494.8-944.8$ & 576.3 & 422.2 & 313.3 & 1820.4 & $* * * \mathrm{C}$ & \\
\hline \multicolumn{10}{|c|}{ HOMA $(\mathrm{pmol} / 1 \times$ mmol/l) } \\
\hline Control & 20 & 6,88 & $4.56-9.20$ & 5.55 & 4.95 & 2.30 & 24.69 & & \multirow{3}{*}{0.0014} \\
\hline Intermediate & 15 & 12,30 & $7.78-16.82$ & 9.73 & 8.16 & 4.36 & 31.94 & $* * \mathrm{C}$ & \\
\hline GDM & 16 & 13,29 & $8.62-17.96$ & 11.95 & 8.76 & 3.63 & 42.50 & $* * * \mathrm{C}$ & \\
\hline \multicolumn{10}{|l|}{ IRI } \\
\hline Control & 20 & 0.68 & $0.57-0.80$ & 0.65 & 0.25 & 0.3 & 1.10 & & \multirow{3}{*}{0.001} \\
\hline Intermediate & 15 & 0.93 & $0.77-1.09$ & 0.93 & 0.29 & 0.31 & 1.38 & & \\
\hline GDM & 16 & 1.67 & $1.29-1.70$ & 1.69 & 0.38 & 0.91 & 1.97 & $* * * \mathrm{C}, * \mathrm{IN}$ & \\
\hline \multicolumn{10}{|l|}{ Visfatin (ng/ml) } \\
\hline Control & 20 & 76.8 & $70.2-83.4$ & 79.2 & 14.1 & 42.5 & 93.8 & & \multirow{3}{*}{0.009} \\
\hline Intermediate & 15 & 84.0 & $75.9-92.2$ & 88.3 & 14.7 & 56.2 & 98.9 & & \\
\hline GDM & 16 & 93.1 & $86.2-99.9$ & 90.3 & 12.3 & 67.0 & 113.3 & $* * \mathrm{C}$ & \\
\hline
\end{tabular}

$C$, compared with control group; IN, compared with intermediate group.

Significant differences in mean values in two groups, assessed by means of a Mann-Whitney test, are indicated by asterisks: ${ }^{*} p \leq 0.05,{ }^{* *} p \leq 0.01$, $* * * p \leq 0.001$.

a $p$ value represents the significance level of the Kruskal-Wallis test for comparison of distributions of these characteristics in three independent groups.

\section{Discussion}

In this paper, we demonstrate an increase in fasting visfatin concentrations with a worsening degree of glucose intolerance, with higher levels in women with GDM (see ESM). During the preparation of our paper, Krzyzanowska et al. [9] reported raised visfatin levels in GDM. In contrast to their data, we found a significant correlation between visfatin and fasting insulin and HOMA. Furthermore, we are the first to demonstrate a significant correlation between visfatin and insulin during the OGTT.

Pregnancy is associated with alterations in insulin resistance and sensitivity, the latter declining with increasing gestation. Given its reported insulin mimetic effects $[1,5]$, visfatin may well counteract the high glucose levels, particularly in GDM. However, the results of our study show that such an interpretation, although theoretically correct, is more complex. First, the variations of insulin and

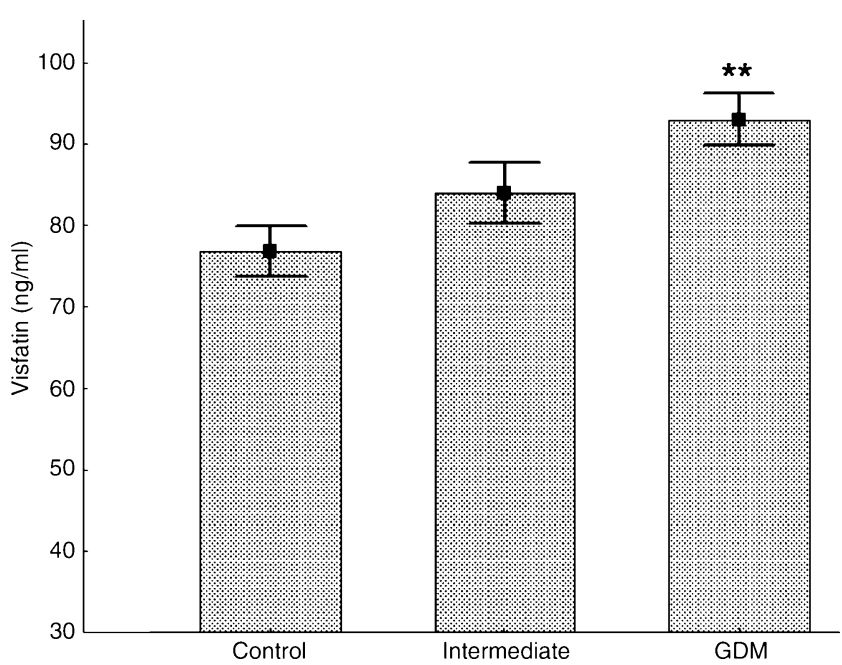

Fig. 1 Visfatin levels (means $\pm \mathrm{SEM}$ ) in control, intermediate (i.e. false-positive GCT) and GDM groups. ${ }^{* *} p<0.01$, significantly higher in comparison with the control group (Mann-Whitney $U$ test) 
glucose explain only $18 \%$ of the variation of serum visfatin in the multivariate model. This implies that other, yet unidentified, factors are likely to determine most of the variation of serum visfatin in pregnancy. Second, there are major differences in the assessment of IR. For example, if one takes into account only fasting glucose and insulin (HOMA), then women with GDM and those in the IG group are more IR than control subjects, with no significant difference between GDM and IG groups. On the other hand, if post-glucose-load glycaemia and insulinaemia are accounted for (IRI), then this reveals a marked difference between the GDM group and both the IG and control groups. Therefore, mathematical models (HOMA vs IRI) used to calculate IR indices in pregnancy may give discrepant results, and thereby a relationship between visfatin and calculated IR indices.

Very recently, unlike our findings and those by Krzyzanowska et al. [9], Chan et al. [10] have reported lower visfatin levels in women of Chinese origin with GDM. The precise reason for these differences is unclear. However, the study design by Chan et al. was different: the time of GDM testing was about 24 instead of 28 weeks; different criteria were used to diagnose GDM; and no fasting or post-OGTT glucose values or insulin resistance parameters were assessed in their study. Furthermore, subjects of Far Eastern origin have significant differences in insulin sensitivity in comparison with subjects of Europid origin [11], and therefore it is not possible to directly compare the results of our study with the study by Chan et al. [10].

Currently the data on a relationship between visfatin and insulin sensitivity in humans are conflicting. Some authors report a lack of correlation between plasma visfatin and insulin/HOMA [2, 4], while others [3], like us [12], have observed a significant correlation between plasma visfatin and fasting insulin and HOMA. Moreover, Haider et al. [13] report a significant decrease of plasma visfatin concentrations in obese subjects after gastric banding, where individual changes in insulin resistance and visfatin were significantly associated $(r=-0.43, p<0.05)$. In addition, in the present study we describe for the first time a significant correlation between visfatin and insulin, and visfatin and glucose concentrations at $120 \mathrm{~min}$ of the OGTT in pregnant women. Given that our subjects with GDM had a more pronounced rise in glucose after OGTT than both IG and control groups, this raises the possibility that higher visfatin levels in GDM could be related to the visfatin response to hyperglycaemia. Such a hypothesis is supported by the results of Haider et al. [14], who demonstrated that glucose infusion (under clamp conditions) could increase visfatin concentrations. Recently, it has also been shown that subjects with established type 2 diabetes have significantly raised visfatin levels when compared with newly diagnosed type 2 diabetic subjects
[15]. These findings are of interest, as GDM, like type 2 diabetes, is characterised by relative hyperglycaemia and the metabolic defects of insulin resistance [16].

Limitations of our study include its cross-sectional design, and therefore to ascertain the association between the worsening of glucose tolerance during the pregnancy paralleled by the raise of visfatin, a longitudinal study design is necessary with additional data such as any correlation with cord blood visfatin and birthweight. Finally, it is unclear as to the precise source of visfatin and the mechanisms regulating its production; it would be useful to test the hypothesis of inflammation and insulin resistance in GDM and their impact on visfatin.

In summary, our study demonstrates significantly higher serum visfatin levels in women with GDM as well as a positive correlation between serum visfatin and both fasting and post-glucose-load insulin. The precise significance of our findings remains to be elucidated, and it remains to be established whether visfatin may also be involved in regulation of insulin sensitivity during human pregnancy.

Duality of interest The authors have no duality of interest with regard to this study.

\section{References}

1. Fukuhara A, Matsuda M, Nishizawa M et al (2005) Visfatin: a protein secreted by visceral fat that mimics the effects of insulin. Science 307:426-430

2. Berndt J, Kloting N, Kralisch S et al (2005) Plasma visfatin concentrations and fat-specific mRNA expression in humans. Diabetes 54:2911-2916

3. Chen MP, Chung FM, Chang DM et al (2006) Elevated plasma level of visfatin/pre-B cell colony-enhancing factor in patients with type 2 diabetes mellitus. J Clin Endocrinol Metab 91:295-299

4. Pagano C, Pilon C, Olivieri M et al (2006) Reduced plasma visfatin/pre B-cell colony enhancing factor in obesity is not related to insulin resistance in humans. J Clin Endocrinol Metab 91:3165-3170

5. Kim M-K, Lee JH, Kim H et al (2006) Crystal structure of visfatin/pre-B cell colony-enhancing factor/nicotinamide phosphoribosyltransferase, free and in complex with the anti-cancer agent FK-866. J Mol Biol 362:66-77

6. WHO consultation (1999) Definition, diagnosis, and classification of diabetes mellitus and its complications. Report of a WHO consultation. Part 1: Diagnosis and classification of diabetes mellitus. WHO/NCD/NCS/99.2. WHO, Geneva

7. Matthews DR, Hosker JP, Rudensky AS, Naylor BA, Treacher DF, Turner RC (1985) Homeostasis model assessment: insulin resistance and beta-cell function from fasting plasma glucose and insulin concentrations in man. Diabetologia 28:412-419

8. Matsuda M, DeFronzo R (1999) Insulin sensitivity indices obtained from oral glucose tolerance testing. Diabetes Care 9:1462-1470

9. Krzyzanowska K, Krugluger W, Mittermayer F et al (2006) Increased visfatin concentrations in women with gestational diabetes mellitus. Clin Sci (Lond) 110:605-609 
10. Chan TF, Chen YL, Lee CH et al (2006) Decreased plasma visfatin concentrations in women with gestational diabetes mellitus. J Soc Gynecol Investig 13:364-367

11. Wijeyaratne $\mathrm{CN}$, Balen AH, Barth JH, Belchetz PE (2002) Clinical manifestations and insulin resistance (IR) in polycystic ovary syndrome (PCOS) among South Asians and Caucasians: is there a difference? Clin Endocrinol (Oxf) $57: 343-350$

12. Tan BK, Chen J, Digby JE et al (2006) Increased visfatin messenger ribonucleic acid and protein levels in adipose tissue and adipocytes in women with polycystic ovary syndrome: parallel increase in plasma visfatin. J Clin Endocrinol Metab 91:5022-5028
13. Haider DG, Schinler K, Schaller G, Prager G, Wolzt M, Ludvik B (2006) Increased plasma visfatin concentrations in morbidly obese subjects are reduced after gastric banding. J Clin Endocrinol Metab 91:1578-1581

14. Haider DG, Schaller G, Kapitis S, Maier C, Luger A, Wolzt M (2006) The release of the adipocytokine visfatin is regulated by glucose and insulin. Diabetologia 49:1909-1914

15. Lopez-Bermejo A, Chico-Julia B, Fernandez-Balsells $\mathrm{M}$ et al (2006) Serum visfatin increases with progressive beta-cell deterioration. Diabetes 55:2871-2875

16. Buchanan TA (2001) Pancreatic B-cell defects in gestational diabetes: implications for the pathogenesis and prevention of type 2 diabetes. J Clin Endocrinol Metab 86:989-993 